\title{
Retraining an editor as a mapmaker
}

A sequence-specific labeling system based on CRISPR/Cas9 enables simultaneous imaging of multiple chromosomal sites in live cells.

CRISPR users typically use single guide RNA (sgRNA) sequences to direct the genome-editing capabilities of the Cas9 enzyme to a specific DNA site. However, University of Massachusetts Medical School researcher Hanhui Ma and colleagues have instead exploited this sequence selectivity in a multiplex fluorescent labeling strategy for visualizing the dynamic behavior of chromosomes. By color-coding as many as six different sites at once, the team was able to observe differences in mobility between chromosomes and even at multiple sites within a single chromosome.

They achieved this by appending three different RNA hairpin structures in various combinations onto sgRNAs designed to target selected sequences in the genome. These were partnered with a mutant derivative of Cas9 that lacks the ability to cleave DNA but remains capable of site-specific chromosomal binding when complexed with an sgRNA. Each hairpin sequence in turn had a cognate RNA-binding protein partner, which was coupled onto a red, green or blue fluorescent protein.

The sgRNA for any given target sequence incorporated a tandem pair of hairpin RNA structures, allowing the researchers to produce many distinct colors. For example, the MS2 hairpin, which is recognized by the blue fluorescent protein fusion, could also be combined with the hairpin sequence that recognizes green fluorescent protein to generate cyan, or with one that recognizes red fluorescent protein to generate magenta.

As an initial demonstration of their approach, dubbed CRISPRainbow, Ma and colleagues showed that they could clearly and accurately label telomeric sequences with each of their color combinations. They subsequently scaled up, using all six color combinations to label multiple genomic sites at once, and observed striking differences in the extent to which these chromosomal segments moved over the course of a ten-second observation period.

Adding one or more color labels-for example, a far-red tag — should make it possible to extend CRISPRainbow to capture data on as many as 15 distinct chromosomal sites. Longer imaging time scales are also feasible, and the authors see their method as a potentially powerful tool for monitoring in vivo nuclear rearrangements during major cellular 'life events' such as differentiation or the run-up to mitotic division.

Michael Eisenstein

\section{RESEARCH PAPERS}

$\mathrm{Ma}$, H. et al. Multiplexed labeling of genomic loci with dCas9 and engineered sgRNAs using CRISPRainbow. Nat. Biotechnol. 34, 528-530 (2016). 